\title{
Imaginario de una comunidad discursiva: representaciones sociales de la tesis de licenciatura según profesores y estudiantes
}

\author{
Geannini Ruiz Ulloa ${ }^{1}$
}

Recibido: 12 de abril de 2018 / Aceptado: 30 de agosto de 2019

Resumen. En esta investigación, se analizan las representaciones sociales de profesores y estudiantes de la carrera de Sociología de la Universidad de Costa Rica sobre el proceso de escritura de la tesis de licenciatura. A partir de una perspectiva etnográfica, se propone un modelo que permite entender el imaginario de una comunidad discursiva. La tesis como género discursivo faculta a los estudiantes el ingreso a su comunidad académica, no obstante, sobre este ritual de paso no se discute su proceso de escritura (causas, estrategias de lectoescritura, problemas de escritura, etcétera) ni los distintos actores sociales que intervienen en él. Asimismo, se retoman los planteamientos sobre escritura académica de las últimas décadas con la finalidad de insistir en la reflexión de las prácticas de lectura y escritura que se desarrollan en las aulas universitarias.

Palabras clave: Escritura académica, Proceso de escritura, Tesis, Imaginario, Representaciones sociales.

\section{[en] Imaginary of a Discursive Community: Social Representations of the Licenciatura Thesis} according to Teachers and Students

\begin{abstract}
In this article, we analyze the social representations of professors and students of the Sociology degree at the University of Costa Rica on the writing process of the undergraduate thesis. From an ethnographic perspective, a model is proposed that allows to understand the imaginary of a discursive community. The thesis as a discursive genre empowers students to enter their academic community. However, this rite of passage does not discuss its writing process (causes, literacy strategies, writing problems, etc.) or the different social actors who intervene in it. Also, the approaches on academic writing of the last decades are reviewed to reflect on the practices of reading and writing that are developed in the university classrooms.

Keywords: Academic writing, Writing process, Thesis, Imaginary, Social representations.
\end{abstract}

[fr] Imaginaire d'une communauté discursive: représentations sociales des enseignants et étudiants autour du mémoire de Licence

Résumé. Dans cette recherche, nous analysons les représentations sociales des enseignants et des étudiants en Sociologie de l'Université du Costa Rica. Au moment de la recollection des données, les étudiants se trouvaient au processus d'écriture de leurs mémoires de Licence, guidés par les enseignants consultés. À partir d'une perspective ethnographique, nous proposons un modèle permettant de comprendre l'imaginaire d'une communauté discursive donnée. Bien que le mémoire académique soit un genre discursif à part entière, permettant aux étudiants d'entrer dans la communauté universitaire, il existe encore peu de recherches concernant son processus d'écriture (causes, stratégies et difficultés de lecture/écriture, etc.) et les différents acteurs sociaux qui interviennent dans ce rite de passage. Aussi, nous visitons les différentes approches développées ces dernières décennies autour de l'écriture universitaire, afin d'apporter une réflexion sur les pratiques de lecture et écriture existantes dans les salles de cours aujourd'hui.

Mots-clés: Écriture universitaire, Processus d'écriture, Mémoire, Imaginaire, Représentations sociales.

Sumario: 1. Introducción. 2. Fundamentación teórica. 3. Antecedentes. 3.1. Estudios internacionales. 3.2. Estudios nacionales. 4. Metodología. 5. Resultados. 5.1. Escritura de los aprendices. 5.2. Escritura de los maestros. 5.3. Imaginario de una comunidad. 6. Limitaciones y conclusiones. 7. Bibliografía.

Cómo citar: Ruiz Ulloa, Geannini (2020): "Imaginario de una comunidad deiscursiva: representaciones sociales de la tesis de licenciatura según profesores y estudiantes”, en Didáctica. Lengua y Literatura, 32, 85-106. 


\section{INTRODUCCIÓN}

¿Problemas de escritura o de lectura en los jóvenes universitarios? ¿Una realidad actual en las aulas universitarias? En el contexto internacional y, particularmente, el costarricense, ha existido una larga tradición de acercamientos sobre la problemática de la escritura y lectura en la educación superior. No obstante, sobre la escritura académica persisten muchas representaciones e ideas que asocian el escribir como una actividad individual y en solitario en donde se valora el manejo del componente gramatical (ortografía, sintaxis, etcétera) antes que uno argumentativo.

En esta visión, los problemas de escritura que pueden experimentar los estudiantes universitarios son deficiencias de una formación previa, por tanto, serán los mismos estudiantes los que deberán atender y buscar posibles soluciones para escribir en la universidad. Esto último ha sido cuestionado por nuevos acercamientos teóricos (Carlino, 2005) que demuestran cómo la universidad también se convierte en un campo de escritura que crea sus propias reglas y conjuntos de textos según los propósitos de las comunidades disciplinares.

Sobre la tesis, texto por antonomasia perteneciente a la academia, se han escrito infinidad de manuales y guías que dictan idealmente fórmulas de escritura para que el estudiante consiga concluirla. Sin embargo, casi nunca se discute la dimensión social de su escritura, es decir, las representaciones que tienen los sujetos cuando escriben, los valores que asocian en relación con el discurso académico y los actores que intervienen en su proceso de escritura.

Esta investigación, desde una perspectiva etnográfica, busca un nuevo acercamiento a la manera en que la tesis es entendida como género discursivo dentro de una comunidad académica particular, en este caso, la carrera de Sociología de la Universidad de Costa Rica. En ese sentido, estudiar las prácticas específicas de escritura y lectura en la universidad nos permite responder a los retos comunicativos que tienen los estudiantes actualmente. Además, de entender las demandas del campo académico en donde la escritura se convierte en el medio más frecuente para el intercambio comunicativo.

\section{FUNDAMENTACIÓN TEÓRICA}

Flowerden (2002) y Bhatia (2012) proponen el abordaje de una dimensión etnográfica en el estudio de la escritura académica, la cual tendría el objetivo de situarnos en la comprensión del contexto en que se produce un texto y la manera en que este es entendido por los sujetos que participan en su construcción. Generalmente, las investigaciones que han seguido esta perspectiva en Hispanoamérica pueden agruparse en dos líneas: representaciones sociales (Marinkovich y colaboradores, 2011-2014) y concepciones (Molano y Stella López 2007; Castelló 2000 y Carlino e Iglesia, 2013).

Por un lado, el concepto de representaciones sociales brinda la oportunidad para reflexionar sobre la manera en que se entiende la realidad y cómo el sujeto la interpreta. Marinkovich y Tapia (2013) indican que las representaciones sociales son producidas por sujetos sociales, y concebidas "como una producción y elaboración de carácter social no impuesta externamente, pues conforman un conocimiento espontáneo, práctico, un producto y proceso de la actividad" (151). También Araya (2002) afirma que "constituyen sistemas cognitivos en los que es posible reconocer la presencia de estereotipos, opiniones, creencias, valores y normas que suelen tener una orientación actitudinal positiva o negativa" (11). Por tanto, las representaciones sociales son entendidas como conocimientos que tienen los sujetos sobre un fenómeno en particular.

Dentro de este acercamiento, el investigador está llamado a entender cómo los sujetos interpretan y valoran el fenómeno o acontecimiento en estudio con la finalidad de explicarlo. En el ámbito de la educación, las representaciones sociales han permitido a docentes e investigadores conocer cómo los estudiantes representan mentalmente lo enseñado en clase (Lacolla, 2005; y Materán, 2008), de ahí que su traslado a la investigación sobre escritura académica haya tenido una recepción positiva².

Asimismo, otro concepto que es necesario examinar es el de discurso académico. Para ello, se retoma los planteamientos de Hyland (2004), quien propone que el discurso académico es una forma privilegiada de argumento en la modernidad que presenta un modelo de racionalidad y de premisas lógicas que se identifican como el discurso de la Verdad. En otras palabras, el discurso académico ofrece una descripción realista del mundo natural y humano que sirve para distinguir la realidad contingente: es una forma de persuasión a la que se confiere una garantía de conocimiento confiable. Por tanto, la característica principal del discurso académico será persuadir a los lectores sobre la validez y pertinencia de una teoría o hipótesis antes que otra. Hyland (2004) también demuestra que según sea la disciplina se producen maneras particulares de discursos y reglas discursivas.

En relación con lo anterior, Swales (2008) plantea seis características que identifican a una comunidad discursiva, a saber: a) está orientada al logro de unos objetivos públicos ampliamente consensuados, b) tiene determinados mecanismos de comunicación entre sus miembros, c) utiliza esos mecanismos de participación para proporcionar información y retroalimentación, d) es poseedora de uno o más géneros para el logro comunicativo de sus fines, e)

Sin embargo, hay que tener en cuenta que el estudio de las representaciones sociales es más complejo y requiere una profundización del espacio social más exhaustivo que lo realizado en investigaciones sobre escritura académica (Araya, 2002). 
junto a los géneros que le son propios dispone de una terminología específica y f) está compuesta por un determinado número de miembros que poseen los conocimientos relevantes y las experiencia discursivas necesarias ${ }^{3}$.

Establecer una comunidad discursiva como referencia permite entender la manera en que dentro de ella se desarrollan los géneros discursivos; pues estos son entendidos como clases de eventos comunicativos. Los miembros de una comunidad discursiva utilizan los géneros discursivos según las metas que tengan, las cuales también son compartidas por otros miembros. En resumen, el discurso académico y sus géneros discursivos son producidos por comunidades discursivas que desarrollan su trabajo en centros de enseñanza superior.

Uno de los géneros discursivos más empleados dentro de las comunidades académicas corresponde a la tesis. Su

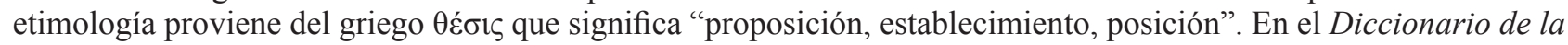
Real Academia Española (DRAE) se encuentran dos acepciones: a) "conclusión, proposición que se mantiene con razonamientos" o b) "disertación escrita que presenta a la universidad el aspirante al título de doctor en una facultad". Esta última nos ubica más hacia al tipo de texto que nos referimos en este trabajo, ya que la tesis es uno de los requisitos fundamentales para la obtención de un título universitario (ya sea licenciatura, maestría o doctorado). Parodi (2008), en su clasificación sobre géneros académicos y géneros profesionales, define la tesis como:

Género discursivo que tiene como macropropósito persuadir acerca de un planteamiento teórico o ideológico. Idealmente, circula en el ámbito científico y la relación entre los participantes es entre escritor experto y lector experto. El modo de organización discursiva predominante es argumentativo. Se suele emplear recursos multimodales $(2008,66)$

El estudio de Bosinger (2005) realiza una descripción de la tesis como una clase textual que se divide en cinco apartados: función, situación comunicativa, procedimientos, estructura textual y fórmulas estilísticas. La tesis presenta distintas funciones; sin embargo, las tres más usuales son: informar sobre los aportes encontrados en la investigación; ingresar a una comunidad académica "la tesis se constituye en un medio para ser aceptado como un par por la comunidad de especialistas" (268); y, por último, persuadir sobre la validez, la relevancia y la originalidad de los resultados del trabajo.

La situación comunicativa de la tesis está "enmarcada institucionalmente en el ámbito académico" (Bosinger, 2005, 268). El comité científico observa el desarrollo de una investigación, la cual será evaluada con el fin de decidir si el trabajo es satisfactorio y cumple con los requisitos solicitados. De ser positiva la respuesta del tribunal, el aspirante consigue el título. Esto generalmente se da en una situación asimétrica, pues el grupo de expertos asume una posición privilegiada y de autoridad ante el aspirante.

En ese sentido, la tesis se construye como un texto cuidadosamente elaborado que emplea un discurso retórico abundante, que ha implicado un proceso de preparación y maduración de lugar y tiempo diferido entre el escritor y lectores. Asimismo, Bosinger (2005) indica que el vocabulario convencional de la disciplina es un elemento compartido entre los distintos participantes.

\section{ANTECEDENTES}

La escritura académica ha sido objeto de estudio de distintas disciplinas, por ejemplo: Psicología, Pedagogía, Sociología y Lingüística. En el presente estudio se parte desde esta última, dentro de la cual ha habido diferentes líneas de investigación: la textual, la aplicada y la sociocognitiva o etnográfica. A continuación, se hace un repaso de los principales trabajos desde la perspectiva sociocognitiva publicados en los últimos años. Además, se revisan las investigaciones que se han efectuado en Costa Rica sobre expresión escrita.

\subsection{Estudios internacionales}

La línea sociocognitiva o etnográfica aborda el estudio de las concepciones y las representaciones sociales presentes el discurso académico -y de los géneros discursivos- según sea el tipo de población (estudiantes y/o profesores). Estos trabajos tienen el interés de contextualizar la manera en que se desarrollan las prácticas de escritura dentro de un espacio específico con el propósito de conocer cómo se representan tareas de escritura, el posicionamiento de la voz del escritor, concepciones de lenguaje y escritura, entre otros aspectos.

Ochoa (2009) analiza cómo la lectura y escritura pueden convertirse en factores que favorecen u obstaculizan el proceso de escritura de una tesis de maestría, lo anterior a partir de las experiencias de tesistas en sus prácticas de lectura y escritura. Por otro lado, Hernández (2009) presenta las dificultades en la escritura de los estudiantes de una maestría en investigación educativa, a partir de su experiencia como docente de dicho posgrado. Mientras que Pereira y Di Stefano (2009) estudian la manera en que se representa la reescritura de una introducción de una tesis de doctorado a partir de un estudio de caso. Las autoras enfatizan en la importancia que debe tener el reconocimiento de la tesis como rito de iniciación. 
Juana Marinkovich, lingüista chilena, ha sido una de las investigadoras más fructíferas en la investigación de representaciones sociales sobre la escritura académica en el ámbito de la tesis de licenciatura. En un periodo de cinco años, se ha acercado a distintas disciplinas y ha estudiado su relación con la escritura. Veamos un recuento.

Marinkovich y Tapia (2011) señalan el desencuentro entre la forma en que los profesores y estudiantes entienden el proceso de escritura de la tesis a través de un estudio de las representaciones sociales en dos carreras del área de Ciencias del Mar. En cambio, en Marinkovich y Salazar (2011) se realiza un acercamiento a las representaciones sociales que tienen los profesores respecto del proceso de escritura de una tesis de licenciatura en la carrera de Historia. Posteriormente, Marikovich y Tapia (2013) abordan las representaciones sociales presentes en las carreras de Periodismo y Trabajo social, utilizando la misma metodología de los trabajos anteriores. Por último, Marinkovich y Córdova (2014) efectúan una investigación en donde buscan acercarse al fenómeno de la escritura según distintas disciplinas con el objetivo de dilucidar el nexo entre objeto de estudio, método y discursos que se plasman en los géneros discursivos.

\subsection{Estudios nacionales}

En este trabajo, partimos de la división que señala Sánchez (2006b) sobre las principales tendencias que han marcado la expresión escrita en Costa Rica, desde 1985 a 2006:

a) según su mayor o menor apego a la gramática normativa tradicional. En esta dirección, se inscriben los trabajos de: Rojas y Umaña (1976), Jiménez (1987), Quesada (1993) y Garita (2001)

b) La que toma un enfoque lingüístico contemporáneo y trata de encontrar las causas, incorporándose así los trabajos de Rodino y Ross (1985), Rodino (1998) y Sánchez Avendaño. (2006b, 238)

A partir de la división que propone Sánchez (2006b) puede observarse dos tendencias: una hacia los estudios gramaticales y otra que se basa en teorizaciones más contemporáneas que intenten explicar los problemas de escritura y no solo señalar un uso erróneo o bárbaro de la lengua. De estas investigaciones, particularmente, nos interesa comentar tres trabajos que tienen una relación con el presente tema.

Rodino y Ross (1985) plantean la hipótesis de que los errores en expresión escrita en los estudiantes pueden explicarse debido a que ocurre una interferencia del código oral en el código escrito. Los lingüistas cuestionan la idea de que los estudiantes desconozcan la lengua nativa por completo; pues para ellos, en realidad, lo que ocurre es que los estudiantes no conocen las variedades o modalidades expresivas ni las circunstancias enunciativas de la lengua que deben usarse según sea el contexto.

La tesis de Jiménez (1987) propone un abordaje a las actitudes de los estudiantes y los profesores sobre la enseñanza del lenguaje en el nivel superior. La hipótesis de Jiménez fue que, después de implementar un curso de escritura en estudiantes de primer año, se consiguió una mejora en la apreciación de la escritura y lectura. Sin embargo, este trabajo presenta algunas incongruencias metodológicas que ponen en duda la validez de los resultados.

Los trabajos del lingüista Sánchez Avendaño realizados desde el 2004 al 2006 ponen en discusión la enseñanza de la expresión escrita en la universidad. Las investigaciones analizan la historiografía de los libros de enseñanza de la expresión escrita (2004a), la manera de enseñar los signos de puntuación y las unidades textuales (2004b), los problemas más frecuentes de redacción de los estudiantes universitarios (2005a), el uso de los conectores desde una perspectiva discursiva (2005b) y la metodología de enseñanza de los cursos de expresión escrita de la Universidad de Costa Rica (2006a).

\section{METODOLOGÍA}

El tipo de investigación de este trabajo es cualitativo y exploratorio, parte del principio de que la realidad social no es una única, sino múltiple -la cual además arraiga una complejidad de significados y símbolos- que por medio de métodos científicos puede ser interpretada (Ruiz, 1999). Para el estudio de las representaciones sociales, generalmente, se utiliza la Teoría Empíricamente Fundamentada (TEF), la cual plantea cuatro etapas de análisis de la información: codificación abierta, codificación axial, codificación selectiva y generación de la teoría.

El instrumento utilizado fue la entrevista a profundidad. Se establecieron dos grupos de participantes: estudiantes de Sociología que cumplieran con tres criterios mínimos (haber concluido la tesis en rango de cinco años 2011-2016, haber realizado la tesis de manera individual y que su primera carrera fuera Sociología) y profesores de Sociología que cumplieran con dos requisitos (haber sido profesor de la carrera en los últimos cinco años y haber sido director o lector de tesis de licenciatura). El trabajo de campo tuvo como resultado la participación de ocho estudiantes y cuatro profesores ${ }^{4}$.

Las preguntas se articularon en dos ejes: formación académica (que busca dar cuenta sobre la manera en que los estudiantes aprenden a leer y escribir previo a la tesis) y cultura disciplinar (que describe el proceso de escritura de la tesis de licenciatura en la carrera de Sociología). En cada uno de ellos, se plantean distintas categorías que fueron ordenadas bajo el siguiente esquema (ver figura 1).

En un primer momento, se planteó la posibilidad de realizar diez entrevistas en cada grupo, sin embargo, no se pudo completar la cifra anteriormente seleccionada, dos de las dificultades más importantes fueron: la falta de un registro de estudiantes graduados por la unidad académica y cierta desconfianza por parte de los docentes sobre la investigación. 


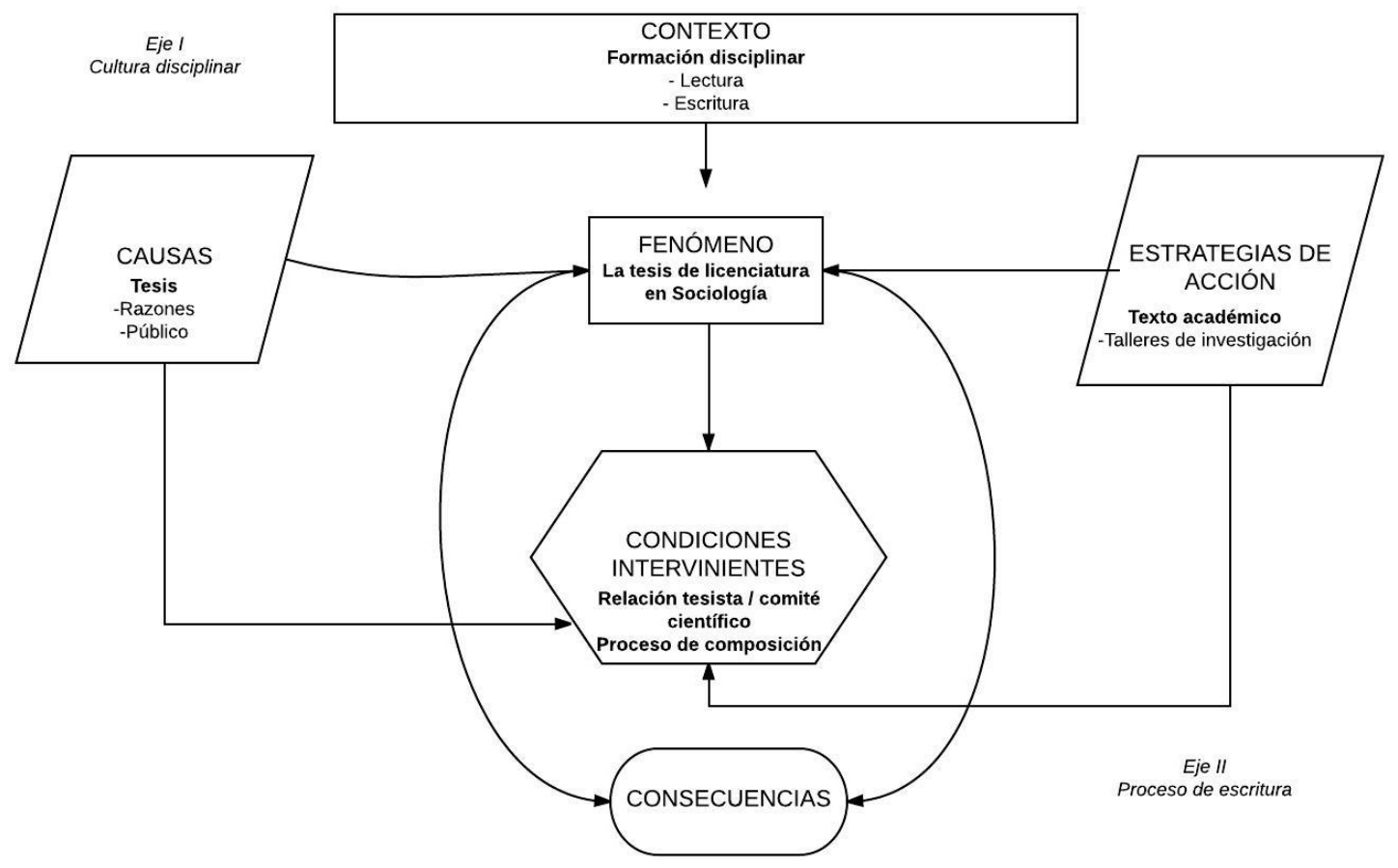

Figura 1. Modelo TEF. Elaboración propia

\section{RESULTADOS}

Los resultados aquí presentados se dividen en tres secciones: escritura de aprendices, escritura de maestros e imaginario de una comunidad. Los dos primeros corresponden a estudiantes y profesores. El tercero es una puesta en común y construcción global de un modelo que permita entender el proceso de escritura de la tesis de licenciatura según los actores de la disciplina.

\subsection{Escritura de los aprendices}

En los primeros años de la carrera, los estudiantes consideraron que la universidad se representa como un campo desconocido para ellos, en el cual debieron entender y aprehender rápidamente las normas y los comportamientos de su carrera con el propósito de ser exitosos. Sin embargo, dicha transición de la educación secundaria a la universitaria no se desarrolló automáticamente y, generalmente, en solitario. En ese sentido, los estudiantes adquirieron una condición de aprendices, tanto de su carrera como del proceso universitario.

La información obtenida en las entrevistas de los estudiantes se ha organizado a partir de las categorías de la TEF (ver figura 1) con el fin de construir un modelo sobre la interacción que tienen los estudiantes en su formación académica. A continuación, discutiremos cada recuadro (ver figura 2).

\section{CONTEXTO \\ -Falta de guía en la escritura y la lectura \\ - Discurso académico desligado de la \\ función social de la carrera}

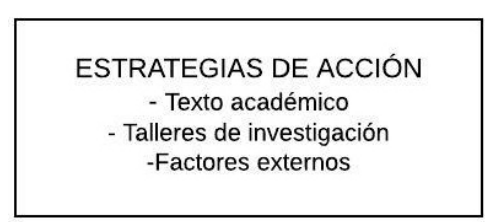

CONDICIONES INTERVINIENTES

Proceso de composición

-Estrategias de lectoescritura

-Relación tesista/ comité científico
CAUSAS

- Obtención del grado

- Interés por la temática

-Compromiso social

TESIS

CONSECUENCIAS

- Visión negativa del impacto real de la tesis

-Ausencia en la preparación de la tesis

Figura 2. Modelo de los estudiantes. Elaboración propia. 
En el caso del contexto, los estudiantes manifestaron una ausencia en la guía de tareas y prácticas de lectura y escritura en el bachillerato. Por ejemplo, en lo correspondiente a los ensayos, los profesores solicitaban en sus cursos la preparación de estos textos sin brindar a los estudiantes los criterios que se utilizarían en la calificación. Semejante ocurre con la lectura, los profesores daban una bibliografía general pero no explican cómo utilizarla y de qué manera los estudiantes podían sacar provecho de los textos referidos. En ese sentido, los estudiantes expresaron sentirse perdidos en muchas tareas que implicaban la interacción con un par académico, pues volviendo al ejemplo de los ensayos, los estudiantes necesitaban saber cuáles eran las características discursivas que sus docentes consideraban imprescindibles para un ensayo académico. Como comenta uno de los entrevistados:

No hay en la escuela algo que te diga cómo hacer un ensayo || yo no sé recuerdo un curso en donde un profesor decía ustedes saben cómo debe ser un ensayo introducción desarrollo y conclusión esa es la estructura que se maneja pero || como ya va más por la formación de cada persona más o menos no hay como \| que se establezca un estilo como de redacción sino más a la libre y ahí ya depende de que tanta experiencia tenés vos como lector qué tantos textos manejas porque ahí eso se permite no sé has leído cien artículos entonces hay algunos que te han como la estructura pero no hay así no sé cómo \| una pauta.

También los estudiantes opinaron que los ejercicios de escritura de la carrera de Sociología estaban más enfocados en las necesidades evaluativas y académicas antes que en una orientación más social que permita vincular la escritura con el quehacer social de los sociólogos, principalmente, fuera del campo universitario ${ }^{5}$.

En el recuadro de estrategias de acción, los estudiantes mostraron tener una postura crítica en relación con el discurso académico y los textos académicos, pues para ellos, el discurso académico se presenta como sectario, ya que que no integra a las comunidades con las que los sociólogos trabajan. Esta representación tuvo una consecuencia en la identificación del público de la tesis, pues, según los estudiantes, la tesis debería ir destinada a las comunidades que participaron en ella.

En el plan de estudios de la carrera, en su cuarto año, los estudiantes de Sociología reciben cursos de investigación que tienen el objetivo de ayudarlos en la preparación del anteproyecto de tesis de licenciatura. Sobre dichos cursos, los estudiantes expresaron que se enfocaban más en el diseño de la investigación y no tanto en la proporción de herramientas para la escritura. Es decir, existe una separación entre la escritura y la investigación, ambas actividades no se entienden de manera complementaria.

En factores externos, los estudiantes de Sociología señalaron la existencia de condiciones que mejoraban o dificultaban el proceso de escritura de la tesis, por ejemplo, contar con una beca para la dedicación exclusiva o tener que trabajar mientras se escribía la tesis. Asimismo, en esta subcategoría, entrarían a colación problemas familiares y personales que afectan el proceso de la tesis.

En condiciones intervinientes, el proceso de composición se dividió en la selección del problema de tesis y la utilización de estrategias de lectoescritura (ver figura 2). En el caso del problema de tesis, este correspondió a un interés o inquietud sobre algún campo de estudio sociológico por parte de los estudiantes durante el bachillerato, cuya consecuencia directa fue la selección del director de tesis, pues tanto profesores y estudiantes compartieron intereses científicos en común previo a la tesis. Paralelamente, los lectores fueron elegidos a partir del grado de cercanía y confianza que los estudiantes experimentaban con ciertos profesores.

En las Estrategias de lectoescritura, sobresale la recursividad como característica del proceso de escritura de la tesis. Por ejemplo, la composición de los borradores de escritura fue sumamente significativo para que los estudiantes pudieran avanzar en la escritura de sus investigaciones. Asimismo, la mayoría manifestó tener dificultades en los procesos de revisión y corrección de sus propios trabajos:

[...] es que yo sí tengo una incapacidad no sé si es la palabra adecuada de $\|$ revisar un texto para detectar errores de escritura me cuesta mucho porque generalmente a veces con textos que son un poco largos uno está cansado ya de escribir y la mente está cansada entonces la revisión que hago generalmente es una revisión muy rápida más que todo una revisión de contenido pero me cuesta leer palabra por palabra para detectar por ejemplo un error que yo cometo muy a menudo es escribir la misma palabra dos veces.

Las principales causas, que influyeron en los estudiantes a realizar el proceso de tesis, fueron principalmente razones laborales. La mayoría de los entrevistados determinó que al contar con un título de licenciatura podrían ser mejor pagados que solo con el de bachillerato. También pesó el deseo de contribuir desde la temática seleccionada y, de esta manera, retribuir a una comunidad lo aprendido en la universidad.

En las consecuencias, se obtuvo una valoración negativa sobre el impacto de la tesis, pues para los estudiantes el lenguaje en que se escribe la tesis está en contradicción con el ethos sociológico. Asimismo, los estudiantes consideraron que la formación disciplinar recibida no fue la suficiente para emprender el proceso de tesis, especialmente, cuando se enfrentaron a la escritura de su investigación. 
Finalmente, se encontró que los estudiantes no solo tienen dificultades del orden formal, es decir, ortográficas o gramaticales; sino que también el plano emocional es un factor determinante para la escritura, el cual no siempre se reconoce en las academias.

\subsection{Escritura de los maestros}

Los profesores de Sociología no solo cumplen con una función pedagógica, sino que, a su vez, se presentan como los maestros de los estudiantes, es decir, son figuras de autoridad que reciben su reconocimiento en relación con los saberes adquiridos que respaldan su posición. Una de sus funciones más importantes es procurar la formación de los futuros sociólogos del país. De la misma manera, que en el apartado anterior, se presentan los resultados obtenidos a partir del análisis de las entrevistas realizadas a los docentes de Sociología (ver figura 3).

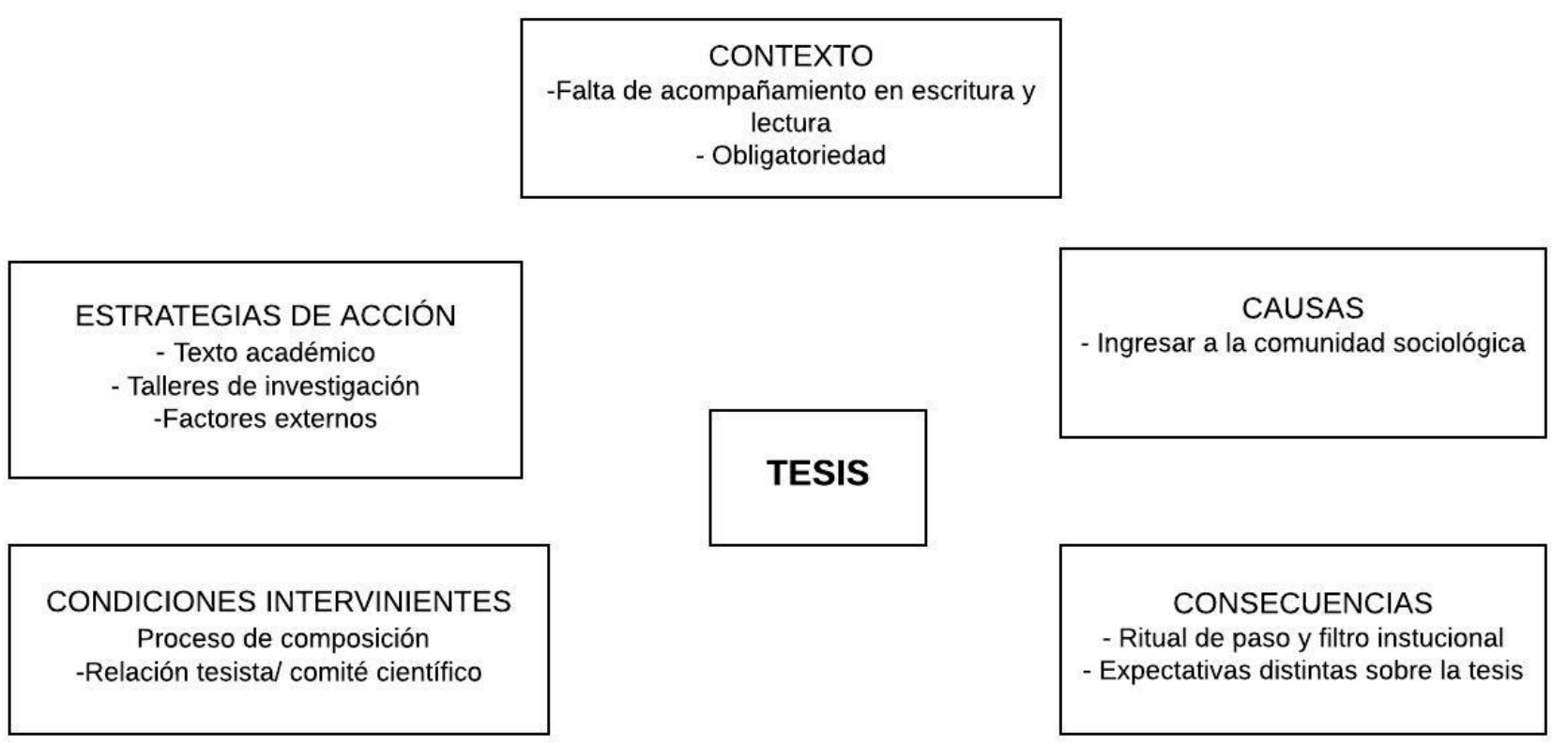

Figura 3. Modelo de los profesores. Elaboración propia

Según el contexto de la carrera de Sociología, cada semestre los profesores desarrollan cursos con una duración de 16 semanas que generalmente buscan situar a los estudiantes dentro de las tradiciones, los modelos y conceptos históricos que ha tenido la Sociología. Ante esto, las prácticas de escritura y lectura son fundamentales para lograr que los estudiantes conozcan y aprendan su disciplina. La mayoría de ellas se desarrolla desde la obligatoriedad, pues son maneras de evaluar el proceso de aprendizaje de los estudiantes.

Ante la recurrencia de problemas de escritura, los profesores se limitan a recomendar cursos de redacción o tímidamente lanzan algunas sugerencias a sus estudiantes, sin embargo, no se consideran lo suficientemente preparados para abordar dentro de sus cursos la escritura y la lectura:

Creo que falta acompañamiento en el para qué leemos yo les digo cuando ustedes están en la carrera van a leer infinidad de artículos de documentos infinidad de textos no solo quédense en el contenido sino vean la forma \| cómo se cita cómo se hace una referencia el libro que está en otro libro o cómo se construye una introducción desarrollo conclusión cosas como muy generales pero que tiene que ver con un ejercicio de distanciarse del texto ver contenido únicamente que es lo que usualmente se les pregunta a observar más allá entonces les digo usted también ahí están aprendiendo no solo del contenido la temática del curso sino están aprendiendo a escribir también porque leer y escribir siempre están de la mano.

En las condiciones intervinientes, el proceso de composición se desarrolla principalmente en la relación que construya el tesista con su comité científico, pues este será el encargado de decidir si cuenta con lo necesario para ingresar a la comunidad de sociólogos. Los docentes señalaron dos perfiles posibles de director: uno que se basa en una relación más cercana con su tesista y otro, menos cercano y que busca darle más autonomía al estudiante. En el caso de los lectores, los profesores opinan que estos mantienen una actitud pasiva, pues respetan la figura y las directrices que brinde el director.

En la escritura de la tesis, los docentes señalaron que la revisión de sus propios textos ayudó a que los estudiantes fueran conscientes de la manera en que expresaban sus ideas. Además, ellos reconocen el componente emocional que interviene en la escritura de la tesis, el cual es ignorado dentro del discurso académico que fomenta un uso impersonal de la escritura (ya que se busca la objetividad científica). 
En las estrategias de acción, los profesores indicaron que los talleres de investigación deberían convertirse en espacios que promuevan una superación de la fragmentariedad que recibieron los estudiantes durante el bachillerato con fin de lograr una integración entre la teoría y la práctica sociológica. Además, opinaron que para esto se requiere una mayor participación de ellos como tutores. También hubo un reconocimiento de otros factores externos que median un proceso de investigación como la tesis: familia, situación económica, trabajo, entre otros.

De acuerdo con los profesores de Sociología, la causa para emprender la tesis de licenciatura es un proceso natural dentro de la comunidad académica. La tesis es un "filtro" que demuestra cuáles estudiantes poseen aptitudes y habilidades necesarias para ser identificados como sociólogos. Esto permite entender que la tesis de licenciatura es un género discursivo que escenifica un ritual de paso, en donde el estudiante atraviesa dos dimensiones: la grupal e institucional, las cuales otorgan el derecho de tener una voz como escritor e investigador.

En el caso de las consecuencias, la ambigüedad de los profesores de Sociología sobre cómo deben actuar en relación con la enseñanza de la escritura y lectura repercute en el hecho de que no exista un consenso sobre una única representación de la tesis de licenciatura. Para los entrevistados, no hay una única representación de la tesis de licenciatura, lo que provoca que muchos estudiantes intenten realizar propuestas que ellos consideran como complejas y pretenciosas. Para los profesores de Sociología, el discurso académico y los textos que se escriben en la universidad tienen un claro destinatario: expertos en la materia. En ese sentido, reconocen que según sea el propósito comunicativo hay un tipo de escritura particular.

\subsection{Imaginario de una comunidad}

Para la construcción del presente modelo de escritura, se puso en diálogo las representaciones de los profesores y estudiantes de Sociología con el fin de explicar el proceso de composición de la tesis de licenciatura (ver figura 4).

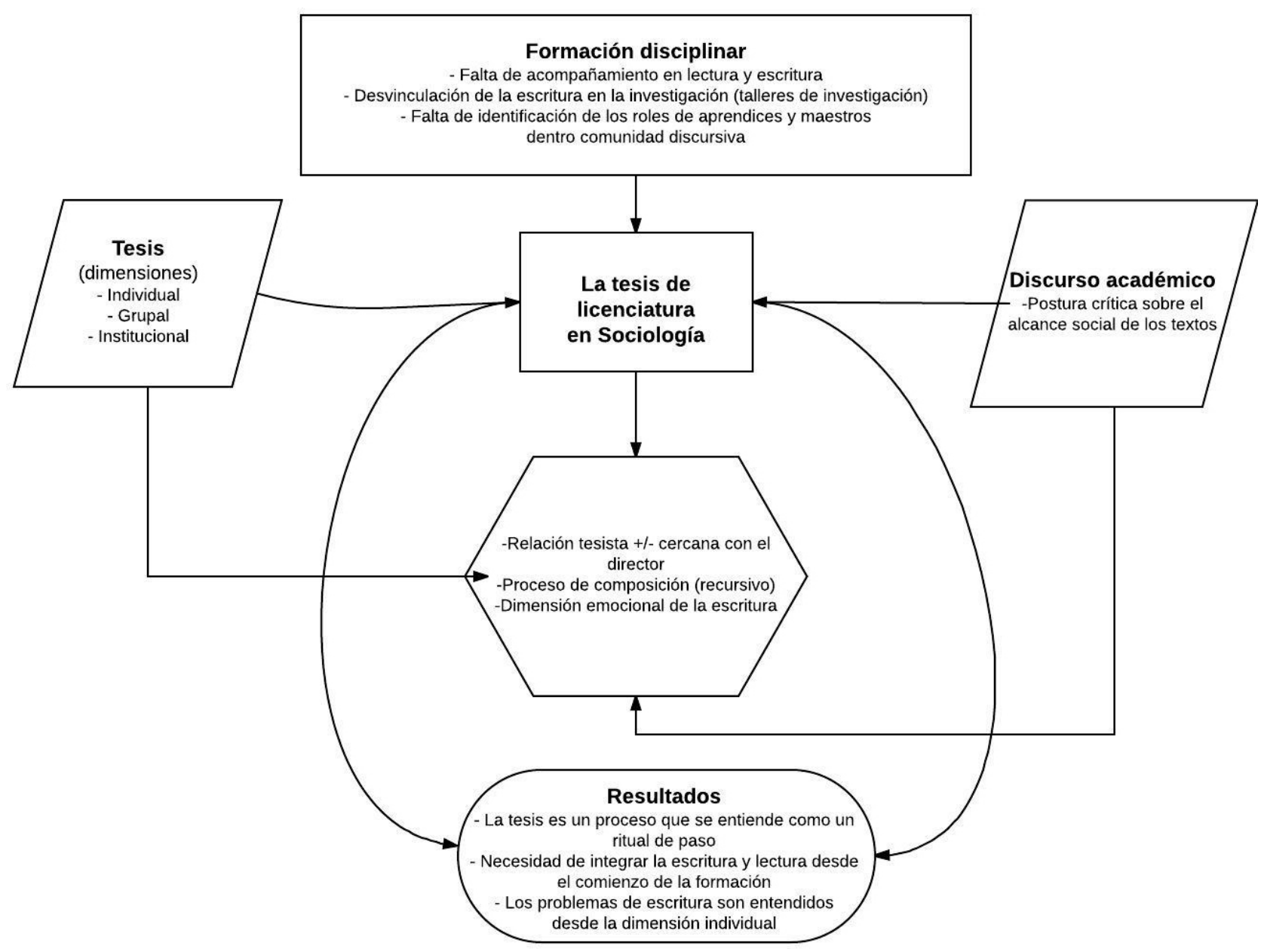

Figura 4. Modelo de la comunidad. Elaboración propia

En el caso de la formación disciplinar, los estudiantes de Sociología experimentaron una falta de acompañamiento en el direccionamiento de las prácticas de lectura y escritura, las cuales se realizaron dentro de una lógica normativa de enseñanza purista-gramatical. En el proceso de escritura de la tesis, algunos de los entrevistados consideraron que la formación recibida en el bachillerato no les brindó las herramientas necesarias para la escritura de la tesis, en ese sentido, cuando ellos tuvieron que enfrentarse a la escritura, se sintieron inseguros al encontrarse en la posición de escritores: 
No nos enseñan a producir desde nuestro contexto elementos teoría e información que sean relevantes para las demás personas en otras palabras a muchos profesores no les importaba lo que teníamos que decir nosotros solo les importaba reproducir en función de ellos lo que sabían de otros autores pero nunca nos dejaron salirnos de alguna forma nos trataban no todos porque hay claras excepciones pero la mayor parte de profesores nos trataban como si no fuéramos capaces de generar ideas $\|$ entonces eso es frustrante a la hora de escribir porque si nadie lo considera a usted y lo digo no solo por mí sino porque hay personas dependiendo del caso la mayor parte de los profesores considerado el ochenta por ciento de los alumnos incapaces de producir su propio material || más allá de un adultocentrismo es como una superioridad intelectual.

Para los profesores de Sociología, la enseñanza de la escritura y lectura dentro de los cursos de carrera es entendida desde dos actitudes. Por un lado, algunos docentes consideraron que la escritura y la lectura son tareas individuales y, por tanto, debe ser una responsabilidad de los estudiantes ocuparse de solucionar sus problemas o dificultades. No obstante, otros de los docentes entrevistados opinaron que también ellos deberían reflexionar más sobre estas materias dentro de sus clases, pues la escritura en la universidad no es la misma que los estudiantes experimentaron en sus colegios. Sin embargo, lo anterior queda a criterio del docente.

En la comunidad científica de la carrera de Sociología, no hay una integración de la lectura y escritura desde la temprana formación, lo cual tiene como consecuencia dos aspectos: una desvinculación de la escritura en la investigación y una falta de identificación en los roles de los maestros/aprendices. En el primer punto, los "Talleres de investigación" (cursos de cuarto año que buscan preparar el anteproyecto de tesis), no cumplen las expectativas de los estudiantes ni de los profesores, generalmente, el curso abarca el aspecto metodológico y muchos estudiantes que llevan el curso ni siquiera trabajan el tema del curso en la tesis, pues solo desean aprobar. En el segundo punto, las representaciones encontradas indican una ausencia en el intercambio abierto de prácticas y conocimientos por parte de los docentes sobre la escritura y la lectura. Para algunos de los estudiantes, la escritura es un símbolo de poder, por tanto, son los expertos de la comunidad quienes poseen solo esa voz.

El discurso académico fue cuestionado por los estudiantes de Sociología, pues lo consideraron hermético al espacio universitario e incomprensible para un público más amplio, debido al uso de un lenguaje técnico y especializado. Los estudiantes reconocieron que para la escritura de la tesis y, especialmente, para su aprobación es necesario reproducir ese uso. Asimismo, la mayoría de los estudiantes entrevistados después de la finalización de su tesis, ha elaborado un producto secundario en relación con su tema, por ejemplo: artículos, documentales o libros con el fin de que su investigación puede ser conocida por un público más amplio ${ }^{6}$.

Para los profesores fue más sencillo reconocer que según sea el tipo de público deseado se debe adaptar el lenguaje. En el caso de la tesis de licenciatura, consideraron que es un texto destinado al comité científico que dictaminará si el estudiante cuenta con las características y condiciones necesarias para ser acreditado como profesional en la disciplina de Sociología. Por tanto, según sea el público destinado, se debe adaptar el lenguaje.

La tesis como ritual de paso es una demostración en tres dimensiones: una individual que se refiere a que el estudiante adquiera su propia voz como escritor-investigador; una grupal, en donde el estudiante es identificado como miembro y par de la comunidad sociológica; y una institucional, en donde el estudiante es certificado como profesional de su disciplina. Cada uno de estos niveles se complementa uno con otro, pues la obtención de la tesis no solo se convierte en un logro personal, sino que representa un avance dentro de la vida académica de los estudiantes.

La relación tesista/comité técnico científico determina el desarrollo de la escritura de la tesis y, consecutivamente, su aprobación dentro de la comunidad discursiva. Sobre la vinculación entre tesistas y directores, Hidalgo y Passarella (2009) demuestran la complejidad e irregularidad que puede darse en dicha relación. Las autoras señalan que es vital fijar las expectativas de los tesistas sobre sus directores desde la primera reunión, pues así se evitan malentendidos y frustraciones con y sobre el director. En el presente caso, los estudiantes hubieran apreciado contar con una disposición de sus directores más próxima, sin embargo, aunque la mayoría de los estudiantes gestionó sus proyectos de tesis de manera individual y autónoma, siempre contó con la aprobación final de sus directores. Según los profesores, la tarea de dirigir una tesis es dar la oportunidad a los estudiantes para que demuestren sus habilidades y atestados en la búsqueda por constituirse como miembros de la comunidad. Tanto profesores como estudiantes coincidieron en la existencia de dos posibles perfiles de director: uno más paternalista y otro que establece una relación de colega.

No obstante, cada relación entre un tesista y director es sumamente diversa, pues los involucrados poseen sus propias características en su carácter y modo de organización; y aunque no es posible generalizar un patrón, si se encuentra evidencia que la vinculación temprana de un estudiante a un campo de la Sociología durante el bachillerato conduce a la identificación de un problema de investigación que generalmente constituye el tema de tesis y que tiene como consecuencia la elección del director, pues se establece un interés en común entre ambos participantes.

De manera similar, en Marinkovich y Salazar (2011), los profesores de Historia consultados afirmaban la necesidad de que los estudiantes se incorporen a temáticas e intereses de investigación previamente dirigidos por ellos como docentes. También Carlino (2003) encuentra que un factor que beneficia la conclusión de la tesis en el posgrado es la vinculación de los tesistas en investigaciones mayores coordinadas por grupos de investigadores, ya que aparte

\footnotetext{
Sobre el público de la tesis, resultó muy interesante ver, por un lado, cómo los estudiantes señalan que la tesis podría ser leída por cualquier persona, señalando la existencia de un lector ideal, no obstante, se reconoce al comité asesor como lector real. Esto provoca cierta insatisfacción en los estudiantes que consideran que los grupos y comunidades que participan en sus trabajos no pueden leer la tesis fácilmente.
} 
del acompañamiento que reciben también es posible que consigan financiamiento que les posibilite la dedicación exclusiva a la investigación.

En el proceso de escritura de la tesis de licenciatura encontramos dos dimensiones en los problemas de los estudiantes: una operacional y una emocional. Para esta investigación, parece importante señalar el componente emocional de la escritura, pues comúnmente no es visualizado debido a que persiste la representación de que la escritura de una tesis es una actividad objetiva y científica. No obstante, profesores y estudiantes apuntaron que el componente emocional en la tesis es un factor significativo, lo cual generalmente no ha sido analizado en otros estudios (Molano y Stella 2007, Ochoa 2009, y Marinkovich y colaboradores 2011-2014). Tanto profesores como estudiantes mantienen una compresión de los problemas de redacción en un ámbito individual. Como lo advierte Becker (1983), el no-reconocimiento de que profesores, investigadores y estudiantes experimentan problemas de redacción obstaculiza la aparición de un diálogo sobre un tema que parece tornarse sombrío y vergonzoso.

Asimismo, se evidencia la necesidad de integrar la escritura y lectura desde el comienzo de la formación disciplinar, pues como han insistido Carlino (2005c), Camps y Milian (2000), y Hernández (2009), la introducción (también llamada alfabetización académica) de las prácticas de escritura y lectura influye en una manera de pensar y comprender la realidad; demostrándose así que la escritura posee una función epistémica más allá de solo ser vista como un instrumento.

\section{LIMITACIONES Y CONCLUSIONES}

Por la naturaleza del presente estudio, los resultados encontrados pueden servir de referentes para futuras investigaciones en otras carreras de Ciencias Sociales, aunque están condicionados a la disciplina de la Sociología. Además, este estudio permitió replantear la discusión sobre la escritura académica en la Universidad de Costa Rica que, desde los últimos trabajos de Sánchez Avendaño (2006), no se había tratado más al respecto.

Al abordar un género académico específico como "la tesis de licenciatura" que, por extensión, es asumido como natural en el desarrollo de la vida académica, se buscó profundizar la manera en que una práctica de escritura es entendida por un grupo académico a partir de las representaciones que influyen en cómo se escribe y a quién se escribe. En ese sentido, se quiso recuperar el valor de la dimensión social y cultural de la escritura como una práctica social e histórica (Chartier 2000). Lo anterior propone una superación en la manera de concebir la escritura: primero, como una actividad individual y solitaria y, segundo, como un arte que solo ciertas personas poseen el don, dejando a los demás mortales excluidos (Cassany 2011). La escritura como práctica social puede desarrollarse en el transcurso del tiempo, ya que su aprendizaje depende de la interacción entre un grupo de sujetos definidos en un espacio delimitado. En otras palabras, aprender a escribir es una manera de formar parte de una comunidad y de una sociedad.

Con nuestro modelo sobre el proceso de escritura (ver figura 4), se buscó integrar más factores y elementos que anteriormente no habían sido valorados, por ejemplo, la relación entre el tesista y su director, los problemas de escritura, entre otros. Con esto, se contradice la idea de que la tesis solo sea pensada como un producto final, pues interesó señalar el proceso que se gesta en la elaboración externa (recopilación y sistematización de un corpus) e interna (planificación y escritura de un texto).

Se comprobó que la tesis de licenciatura es un género discursivo que posee una función social, la cual consiste en una demostración del conocimiento acumulado por los estudiantes en sus años de formación disciplinar. Es decir, que el valor de la tesis de licenciatura recae en que los estudiantes demuestren haber aprendido las habilidades y los conocimientos necesarios para presentar, en ese texto, llamado tesis, una disciplina y solidez metodológica que permita otorgarles el título profesional de sociólogos. Con esto, no se niega la posibilidad de que los estudiantes aporten nuevos conocimientos o teorías, sin embargo, es por medio de esa demostración que, a su vez, el estudiante adquiere una autoridad y una voz en su comunidad discursiva.

A pesar de que solo se describieron los problemas de escritura que tuvieron los estudiantes de Sociología, esta investigación fue un primer intento para acercarse en otra dirección, más allá del señalamiento patológico-gramatical (Rodino y Ross, 1985) a los problemas escritura, pues se evidencia que el elemento emocional también está presente en la escritura académica -sobre todo en la tesis- circunstancia de la que casi nunca se habla, pero que tanto profesores y estudiantes universitarios han experimentado.

\section{BIBLIOGRAFÍA}

Araya, Sandra (2002): Las representaciones sociales: ejes teóricos para su discusión, San José, Editorial de la Universidad de Costa Rica.

Bhatia, Vijay (2002): “A Generic View of Academic Discourse” en Academic Discourse, John Flowerdew (ed.), Londres, Pearson Education.

Becker, Howard (2011): Manual de escritura para cientificos sociales, Buenos Aires, Siglo XXI.

Becher, Tony (2001): Tribus y territorios académicos. La indagación intelectual y las culturas de las disciplinas, Barcelona, Editorial Gedisa. 
Bourdieu, Pierre (2008): Homo academicus, Buenos Aires, Siglo XXI.

Bosinger, Ann (2005): "La tesis", en Los textos de la ciencia: principales clases del discurso académico-científico, Liliana Cubo de Severino (ed.), Córdoba (Argentina), Editorial Comunicarte.

Camps, A. y M. Milian (2000): El papel de la actividad metalingüistica en el aprendizaje de la escritura, Buenos Aires, Ediciones Homo Sapiens.

Carlino, Paula (2005): Escribir, leer y aprender en la universidad: Una introducción a la alfabetización académica, Buenos Aires, Fondo de Cultura Económica.

Castelló, Monserrat (2000): “Las concepciones de los estudiantes en la escritura académica”, en El papel de la actividad metalingüística en el aprendizaje de la escritura, A. Camps y M. Milian (eds.), Buenos Aires, Ediciones Homo Sapiens.

Castelló, Montserrat (coord., 2012): Escribir y comunicarse en contextos científicos y académicos, Barcelona, Editorial Graó.

Chatier, Roger (2000): Cultura escrita, literatura e historia, México D.F, Fondo de Cultura Económica, $2^{\mathrm{a}}$ ed.

Flowerdew, John. (2002): "Ethnographically inspired approaches to the study of academic discourse", en Academic Discourse, John Flowerdew (ed.), Londres, Pearson Education.

Hérnandez, Gregorio (2009): “Escritura académica y formación de profesores. ¿Por qué no acaban la tesis?”, en Tiempo de educar, 10 (19), 11-40. Http://www.redalyc.org/pdf/311/31113164002.pdf [consulta: 14 de junio de 2015].

Hidalgo, Cecilia y Virginia Passarella (2009): "Directores y tesistas: una relación compleja", en Escritura y producción de conocimiento en carreras de posgrado, Elvira Narvaja (ed.), Buenos Aires, Santiago Arcos.

Hyland, Ken (2004): Disciplinary Discourses: social interactions in academic writing, Michingan, University of Michigan Press.

Jiménez, Claudio (1987): Un aporte al mejoramiento de la expresión escrita. Tesis para optar al título de licenciado en Filología Española, Universidad de Costa Rica.

Lacolla, Leda (2005): "Representaciones sociales: una manera de entender las ideas de nuestros alumnos", en Revista Electrónica de la Red de Investigación Educativa, 1 (3), 1-17. Http://revista.iered.org [consulta: 17 de junio de 2015 ].

Marinkovich, Juana y Mónica Tapia (2011): "Representaciones sociales sobre la escritura de la tesis en la formación académica inicial en el área de la Ciencias del Mar”, en Revista Onomazein, 24 (2), 273-297. Http://www.redalyc.org/ articulo.oa?id=134522498012 [consulta: 17 de junio de 2015].

Marinkovich, Juana y Mónica Tapia (2013): "Representaciones sociales sobre la escritura de la tesis en dos carreras del área de humanidades: Periodismo y Trabajo Social”, en Revista Belo Horizonte, 13 (1), 145-169. Http://www.scielo. br/pdf/rbla/v13n1/v13n1a08.pdf [consulta: 17 de junio de 2015].

Marinkovich, Juana y Juan Salazar (2011): "Representaciones sociales de proceso de escritura académica: el caso de la tesis en la Licenciatura de Historia”, en Revista Estudio y Pedagogía 37 (1), 84-105. Http://www.scielo.cl/scielo. php?script=sci_arttext\&pid=S0718-07052011000100005 [consulta: 17 de junio de 2015].

Marinkovich, Juana y Alejandro Córdova (2014): "La escritura en la universidad: objeto de estudio, método y discurso", en Revista Signos, 47 (87), 40-63. Http://www.scielo.cl/scielo.php?script=sci_arttext\&pid=S0718093420140001000 03\&lng=es\&nrm=iso\&tlng=en [consulta: 17 de junio de 2015].

Materán, Angie. (2008): "Las representaciones sociales: un referente teórico para la investigación educativa", en Geoenseñanza, 13 (2), 243-248. Http://www.redalyc.org/pdf/360/36021230010.pdf [consulta: 19 de junio de 2015].

Molano, Ligni y Gladys Stella (2007): "Concepciones de profesores y estudiantes sobre la escritura académica en la Universidad de Ucesi”, en Revista Lenguajes, 35(1), 119-146. Http://media.utp.edu.co/referencias-bibliograficas/ uploads/referencias/articulo/823-concepciones-de-profesores-y-estudiantes-sobre-la-escritura-academica-en-launiversidad-icesipdf-vrIt1-articulo.pdf [consulta: 14 de junio de 2015].

Ochoa, Ligia (2009): "La lectura y la escritura en las tesis de maestría”, en Forma y Función, 22 (2), 99-113.

Parodi, Giovanni (ed., 2008): Géneros académicos y géneros profesionales: accesos discursivos para saber y hacer, Valparaíso (Chile), Ediciones Universitarias de Valparaíso.

Pereira, Cecilia y Mariana di Stefano (2007): "El taller de escritura en posgrado: Representaciones sociales e interacción entre pares", en Revista Signos, 40 (64), 405-430. Http://www.scielo.cl/scielo.php?script=sci_arttext\&pid =S0718-09342007000200007 [consulta: 22 de agosto de 2015].

Pereira, Cecilia y Mariana Stefano (2009): "Representaciones acerca de la tesis doctoral en las reescrituras de la introducción un caso de estudio", en Escritura y producción de conocimiento en las carreras de posgrado, Elvira Narvaja (ed.), Buenos Aires, Santiago Arcos Editor.

Rodino, Ana y Ronald Ross (1985): Los problemas de expresión escrita, San José, Editorial UNED.

Ruiz, Juan. (2012): Metodología de la investigación cualitativa, Bilbao (España), Universidad de Deusto, $5^{\mathrm{a}}$ ed.

Sampieri, Roberto, Carlos Fernández y Pilar Baptista (2005): Metodología de la investigación, México, Editorial McGraw Hill.

Sánchez, Carlos (2004): "Historiografía de la enseñanza de la redacción en Costa Rica: Los libros de texto", en Revista de Filología y Lingüística, 30 (1), 219-246. Http://revistas.ucr.ac.cr/index.php/filyling/article/view/4463; https://doi. org/10.15517/rfl.v30i1.4463 [consulta: 22 de agosto de 2015].

Sánchez, Carlos (2004b): "La puntuación y las unidades textuales: una perspectiva discursiva para el estudio de los problemas de su uso y de su enseñanza”, en Revista Educación, 28 (2), 233-254. Http://revistas.ucr.ac.cr/index.php/ educacion/article/view/2262; https://doi.org/10.15517/revedu.v28i2.2262 [consulta: 22 de agosto de 2015]. 
Sánchez, Carlos (2005a): "Los problemas de redacción de los estudiantes costarricenses: una propuesta de revisión desde la lingüística del texto", en Revista de Filología y Lingüística, 31 (1), 267-295. Http://revistas.ucr.ac.cr/index.php/ filyling/article/view/4419; https://doi.org/10.15517/rfl.v31i1.4419 [consulta: 22 de agosto de 2015].

Sánchez, Carlos (2005b): "Los conectores discursivos: su empleo en redacciones de estudiantes universitarios costarricenses", en Revista de Filología y Lingüistica, 31 (2), 169-199. Http://revistas.ucr.ac.cr/index.php/filyling/ article/view/4430; https://doi.org/10.15517/rfl.v31i2.4430 [consulta: 22 de agosto de 2015].

Sánchez, Carlos (2006a): “¿Cuestión de método? Sobre los cursos remediales universitarios de expresión escrita”, en Revista Educación 30 (1), 65-81. Http://revistas.ucr.ac.cr/index.php/educacion/article/view/1795; https://doi. org/10.15517/revedu.v30i1.1795 [consulta: 22 de agosto de 2015].

Sánchez, Carlos (2006b): "Historia de un desencuentro: investigación y enseñanza en la redacción en Costa Rica", en Revista de Filología y Lingüistica, 32 (1), 223-245. Http://revistas.ucr.ac.cr/index.php/filyling/article/view/4329; https://doi.org/10.15517/rfl.v32i1.4329 [consulta: 22 de agosto de 2015].

Swales, John (2008): Genre Analysis. English in academic and research settings, New York, Editorial Cambrigde University Press, $13^{\text {th }}$ ed. 